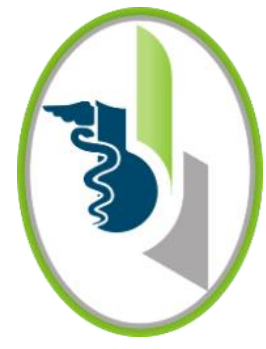

ACCESO 2 ABIERTO

Para citaciones: Cogollo, M., Lujan, M. (2021). Reacción leucemoide asociada con el síndrome de Weil, reporte de caso. Revista Ciencias Biomédicas, 10(2), 145149. https://doi.org/10.32997/rcb-2021$\underline{3375}$

Recibido: 12 de diciembre de 2020 Aprobado: 25 de febrero de 2021

Autor de correspondencia: Marysabel Cogollo González

Editor: Inés Benedetti. Universidad de Cartagena-Colombia. marysabel088@hotmail.com

\section{Reacción leucemoide asociada con el síndrome de Weil, reporte de caso}

\author{
Leukemoid reaction associated with Weil's syndrome, case \\ report
}

\author{
Marysabel Cogollo Gonzalez ${ }^{1}$ (D), Marco Lujan Agámez²
}

${ }^{1}$ Especialización en Neumología, Facultad de Medicina, Universidad de la Sabana, Bogotá, Colombia.

${ }^{2}$ Departamento de Medicina Interna, Facultad de Medicina, Universidad de Cartagena, Cartagena,

Colombia. Departamento de Medicina Interna. Hospital Universitario del Caribe, Cartagena, Colombia.

\section{RESUMEN}

Introducción: La leptospirosis, es una zoonosis de distribución mundial, principalmente en regiones tropicales. El síndrome de Weil, es la forma severa de la leptospirosis, representa el 5-10\% de todos los casos, dentro de las alteraciones hematológicas que lo acompañan, se puede evidenciar una reacción leucemoide, la cual es un hallazgo infrecuente en esta entidad.

Caso clínico: Se describe el caso de un paciente con síntomas sugestivos de síndrome de Weil, quien, en el curso de la hospitalización, desarrolla una reacción leucemoide. Se realizó serología seriada por ELISA para Leptospira la cual fue positiva. Después de la instauración del tratamiento antibiótico, se normaliza el recuento de leucocitos, con egreso hospitalario satisfactorio.

Conclusión: Se debe considerar la presencia de reacción leucemoide en el curso de una leptospirosis severa, para evitar la realización de procedimientos hematológicos innecesarios.

Palabras Clave: leptospirosis; reacción leucemoide; zoonosis.

\section{ABSTRACT}

Introduction: Leptospirosis is a zoonosis with a worldwide distribution, mainly in tropical regions. Weil syndrome, is the severe form of leptospirosis, represents 5$10 \%$ of all cases, within the hematological alterations that accompany it, a leukemoid reaction can be evidenced, which is uncommon an uncommon finding in this entity.

Copyright: (C) 2021. Cogollo, M., Lujan, M. Este es un artículo de acceso abierto, distribuido bajo los términos de la licencia https://creativecommons.org/licenses/by-nc-

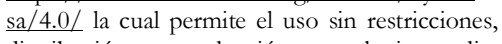
distribución y reproducción en cualquier medio, siempre y cuando el original, el autor y la fuente sean acreditados.

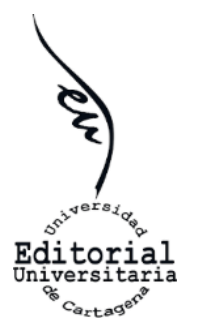

Clinic case: We describe the case of a patient, who admitted to the emergency department with signs and symptoms suggestive of Weil's syndrome, and in the course of hospitalization, he developed a leukemoid reaction. ELISA for Leptospira was positive. He received antibiotic treatment and the leukocytes were subsequently normalized, with satisfactory hospital discharge.

Conclusions: It is important, to consider the presence of leukemoid reaction in the course of severe leptospirosis, to avoid unnecessary hematological procedures.

Keywords: leptospirosis; leukemoid reaction; zoonosis. 


\section{INTRODUCCIÓN}

La leptospirosis, es una zoonosis de distribución mundial, principalmente en regiones tropicales, con mayor impacto en poblaciones con falta de programas de vigilancia en salud pública $(1,2)$. Causa 1.03 millones de casos anuales en todo el mundo (2), con una incidencia en América de 12.5 casos por 100000 habitantes. En Colombia, constituye una entidad de notificación obligatoria, sin embargo, se desconoce la situación epidemiológica exacta, puesto que las publicaciones provienen de estudios de seroprevalencia en pequeñas poblaciones (3). En el registro de vigilancia nacional del 2020, se reportó una incidencia de 0.13 casos por 100000 habitantes (4), existiendo un subregistro, debido a las limitaciones en las pruebas serológicas, escasez de centros especializados que confirmen la patología, y la clínica inespecífica $(5,6)$.

El síndrome de Weil, es la forma severa de la leptospirosis, representa el 5-10\% de todos los casos (7), se caracteriza por disfunción multiorgánica y puede presentarse con fiebre, ictericia, insuficiencia renal, necrosis hepática, afectación pulmonar, colapso cardiovascular, cambios neurológicos y diátesis hemorrágica (8). Dentro de las alteraciones hematológicas, se puede evidenciar una reacción leucemoide (RL), la cual es infrecuente en esta entidad (7). El objetivo del presente trabajo, es describir un caso de un hombre quien desarrolla RL en el curso de una leptospirosis severa.

\section{CASO CLÍNICO}

Sujeto masculino de 48 años de edad, procedente de la ciudad de Cartagena, Colombia. Acude a urgencias, por cuadro de 6 días de fiebre, conjuntivitis, cefalea, náuseas, vómitos, dolor en las pantorrillas e ictericia, sin antecedentes médicos, refiriendo contacto con ratas en su vivienda, además de un cuadro similar en un residente del barrio donde habita. Al examen físico, presión arterial: 110/70 $\mathrm{mmHg}$, frecuencia cardiaca: 92 latidos/minuto, frecuencia respiratoria: 20 respiraciones/minuto, conjuntivitis y escleras ictéricas. Paraclínicos con leucocitosis $\quad\left(25740 / \mathrm{mm}^{3}\right), \quad$ trombocitopenia, hiperbilirrubinemia a expensas de la bilirrubina directa, niveles elevados de creatinina fosfoquinasa (CPK) y lesión renal aguda (tabla 1). Se consideró síndrome febril ictérico-hemorrágico, posible enfermedad de Weil, con inmunoglobulina M (IgM) por ensayo por inmunoabsorción ligado a enzimas (ELISA) para Leptospira, serología para hepatitis, virus de la inmunodeficiencia humana y dengue negativos, se indicó manejo con ceftriaxona y líquidos endovenosos, además de noradrenalina por progreso a choque séptico. Al tercer día, se realiza segundo control de IgM por ELISA para Leptospira, siendo negativa. Presenta mejoría de los azoados, normalización de los niveles de CPK, con destete de vasoactivo. No obstante, persiste con patrón colestásico, y aumento en los leucocitos hasta $59640 / \mathrm{mm}^{3}$, considerándose RL. Extendido de sangre periférica, evidencia células inmaduras y ausencia de blastos, estudios imagenológicos descartan presencia de masas, así como de obstrucción en la vía biliar, y los cultivos son negativos, se cambia antimicrobiano por ampicilina/sulbactam, y se consideró la posibilidad de enfermedad hematológica, solicitando citometría de flujo y valoración por hematología para realización de biopsia de medula ósea, sin embargo se toma una tercera muestra de IgM por ELISA para Leptospira, al octavo día de estancia hospitalaria, la cual salió positiva. Confirmada en una cuarta muestra. El paciente continuó con los antibióticos y líquidos endovenosos, se suspendieron los estudios hematológicos, y en los laboratorios control se observó normalización de los leucocitos y mejoría de la colestasis (tabla 1), con posterior egreso hospitalario.

\section{DISCUSIÓN}

El presente caso, observamos un paciente quien presenta RL, teniendo antecedentes epidemiológicos y clínica compatible con síndrome de Weil, con serología positiva para Leptospira en la segunda semana de haber iniciado los síntomas, no se realizó confirmación con microaglutinación (MAT), ni con técnicas moleculares, debido a la falta de disponibilidad en nuestro hospital. No es necesario, tener el hallazgo del patógeno, para iniciar 
tratamiento antibiótico, es suficiente con la sospecha clínica (6).

Tabla 1. Evolución de los paraclínicos durante la hospitalización

\begin{tabular}{lcccccccc}
\hline \multicolumn{1}{c}{ Parámetro } & Día 1 & Día 3 & Día 5 & Día 7 & Día 10 & Día 13 & Día 15 & $\begin{array}{l}\text { Valores de } \\
\text { referencia }\end{array}$ \\
\hline Hemoglobina & 8.8 & 9.8 & 10.3 & 9.7 & 9.4 & 9.2 & 9.5 & $13.8-17.2 \mathrm{gr} / \mathrm{dl}$ \\
\hline Leucocitos & 25740 & 20350 & 29130 & 59640 & 37830 & 11620 & 10870 & $\begin{array}{l}4500- \\
11000 / \mathrm{mm}^{3}\end{array}$ \\
& & & & & & & & $70-60 \%$ \\
\hline Neutrófilos & 94 & 82 & 70 & 90 & 71 & 87 & 70 & $40-600$ \\
\hline Plaquetas & 61000 & 73000 & 162000 & 270000 & 460000 & 567000 & 528000 & $150000-$ \\
& & & & & & & & $400000 / \mathrm{mm}^{3}$ \\
\hline Creatinina & 5.04 & 3.65 & 0.92 & 1.85 & 1.34 & 1.27 & 1.07 & $0.7-1.3 \mathrm{mg} / \mathrm{dl}$ \\
\hline BUN & 70.6 & 72.5 & 44.1 & 31.02 & 18.7 & 15.56 & 11.25 & $6-20 \mathrm{mg} / \mathrm{dl}$ \\
\hline B. Total & 15.8 & 26.6 & 39.77 & 35.12 & 9.51 & 7.48 & 5.0 & $0.3-1.9 \mathrm{mg} / \mathrm{dl}$ \\
\hline B. Directa & 12.7 & 20.6 & 29 & 26.5 & 8.31 & 6.8 & 4.2 & $0-0.3 \mathrm{mg} / \mathrm{dl}$ \\
\hline CPK & 2143 & 1574 & 314 & - & 97,59 & - & - & $0-195 \mathrm{UI} / \mathrm{L}$ \\
\hline
\end{tabular}

CPK: Creatinina fosfoquinasa; B. Total: Bilirrubina total; B. Directa: bilirrubina directa

El diagnóstico de leptospirosis, se obtiene a través del aislamiento del germen en cultivos, visualización directa en muestras o determinación de ácido desoxirribonucleico (ADN) por proteína $\mathrm{C}$ reactiva (PCR), asimismo con la presencia de anticuerpos, siendo ésta la forma más frecuente de diagnosticar la enfermedad, debido a la baja sensibilidad del cultivo y los altos costos de las técnicas moleculares $(6,9,10)$. La MAT de muestras pareada (con realización de la segunda muestra a los 10-15 días de la primera), permite la confirmación de caso con títulos iguales o mayores a 1:200 (11), aumento de 4 veces en el título de MAT entre muestras de suero agudo y convaleciente o la presencia única de MAT $>1: 400$ (o $\geq 1: 100$ en regiones no endémicas) (6), sin embargo, es una técnica con limitaciones, debido a las diferentes serovariedades presentes en un área geográfica (11), además de ser muy laboriosa, lo que restringe su uso a laboratorios especializados $(5,6)$. Por su parte, la detección de IgM por ELISA, es una prueba costo efectiva, con la desventaja que puede ser negativa en la primera semana (12). Estudios demuestran, que la sensibilidad de esta prueba, oscila entre $38.5-52.7 \%$ en la etapa aguda, en comparación con la sensibilidad de $48.7 \%$ para MAT, en los sueros convalecientes varió de 67.2 a
$84.4 \%$ en comparación con $93 \%$ para MAT (13). En otro estudio, se encontró que la sensibilidad para 2 técnicas de inmunoensayos versus PCR fue de $35 \%$, $30 \%$ y $42 \%$ en los primeros 5 días, respectivamente. Sin embargo, entre el día 6 y 10, las sensibilidades aumentaron a $86 \%, 76 \%$ y $67 \%$, respectivamente (14).

La RL se define como una leucocitosis neutrofílica reactiva, con un recuento de glóbulos blancos superior a $50000 / \mathrm{mm}^{3}$, cuando la etiología es distinta a leucemia. Las principales causas son las infecciones graves (por Clostridium difficile, tuberculosis diseminada, shigelosis severa), neoplasias malignas (linfoma de Hodgkin, melanoma, sarcoma y carcinoma de pulmón, orofaríngeo, gastrointestinal y genitourinario), fármacos (corticoides, minociclina y factores de crecimiento hematopoyético), intoxicaciones (etilenglicol), hemorragia grave o hemólisis aguda (15).

La leptospirosis generalmente cursa con leucocitos normales o ligeramente elevados (16). Son pocos los casos descritos de RL asociado a síndrome de Weil, la literatura lo reporta en el sexo masculino, tanto en 
niños, como en personas adultas, algunos con evolución hacia la mejoría, y otros con curso fatal (8, 17-19).

La RL, se presenta por; a) estimulación de la médula ósea por agentes físicos, químicos o alérgicos; (b) respuesta de la médula ósea a una abrumadora demanda de leucocitos; y (c) hematopoyesis ectópica, por destrucción o invasión del espacio de la médula ósea (20). La presencia de Leptospira en la medula ósea, sugiere que es un microorganismo capaz de atravesar las membranas de hasta $0.22 \mathrm{~mm}$, comportándose como un virus filtrable, que, por diferentes mecanismos, puede destruir células hematopoyéticas, producir oncogenes, así como, mutaciones repetidas, que podrían conducir a la neoplasia y/o desorganizar el genoma de la célula huésped, capaz de producir síndromes leucemoides, que pueden simular leucemias y agravar el estado del paciente (21).

\section{CONCLUSIONES}

La RL se puede presentar en el síndrome de Weil, ante la sospecha clínica, se debe realizar diagnósticos diferenciales con otras entidades, estudios de confirmación para la leptospirosis, con MAT, PCR o cultivo, sin embargo, en zonas donde existe limitaciones, la detección de IgM por ELISA de forma seriada, podría ser una opción, que aunada a los antecedentes epidemiológicos, clínica y evolución del paciente, nos ayuda al diagnóstico de la enfermedad, para un manejo oportuno, evitando la realización de estudios hematológicos innecesarios.

\section{CONTRIBUCIONES DE LOS AUTORES}

Concepción y diseño del estudio: MCG, MLA. Recolección análisis e interpretación de datos: MCG, MLA. Redacción del borrador del artículo, revisión crítica y aprobación de versión final, responsable de la veracidad e integridad del artículo: MCG, MLA.

CONFLICTOS DE INTERESES: ninguno que declarar.

\section{REFERENCIAS}

1. George T, Pais MLJ, Adnan M, Pereira R, Kakribettu RP, Baliga MS. Clinicolaboratory profile of leptospirosis: observations from a tertiary care hospital. J Appl Hematol. 2020; 11: 102- 107.

2. Costa F, Hagan JE, Calcagno J, Kane M, Torgerson P, Martínez-Silveira MS, et al. AI Global Morbidity and Mortality of Leptospirosis: A Systematic Review. PLoS Negl Trop Dis. 2015; 9(9): e0003898.

3. Instituto Nacional de Salud. Protocolo de Vigilancia en Salud Pública. Leptospirosis. [Online]. 2017 Sep 19; Available from: http://www.ins.gov.co

4. Instituto Nacional de Salud. Boletín epidemiológico semana epidemiológica 15 de 2020 [Online]. 2020 Dic 20; Available from: http://www.ins.gov.co

5. Picardeau M. Diagnosis and epidemiology of leptospirosis. Med Mal Infect. 2013; 43: 1-9.

6. Musso D, La Scola B. Laboratory diagnosis of leptospirosis: a challenge. J Microbiol Immunol Infect. 2013; 46: 245-52.

7. Leung J, Schiffer J. Feverish, jaundiced. Am J Med. 2009; 122: 129-31.

8. Maroun E, Kushawaha A, El-Charabaty E, Mobarakai N, El-Sayegh S. Fulminant Leptospirosis (Weil's disease) in an urban setting as an overlooked cause of multiorgan failure: a case report. J Med Case Rep. 2011; 5: 1-4.

9. Haake DA, Levett PN. Leptospirosis in humans. Curr Top Microbiol Immunol. 2015; 387: 65-97.

10. Romero-Vivas CM, Falconar AK. Leptospira spp. y leptospirosis humana. Salud Uninorte. Barranquilla (Col.) 2016; 32: 123-143.

11. Cortés JA, Romero Moreno LF, Aguirre Leon CA, Pinzon Lozano L, Cuervo SI. Clinical approach to acute febrile syndrome in Colombia. Infect. [Online]. 2017 Sep 19; Available from: http://www.scielo.org.co/scielo.php?script=sci arttext\& pid=S0123-93922017000100039\&lng=en

12. Hartskeerl RA, Collares-Pereira M, Ellis WA. Emergence, control and reemerging leptospirosis: dynamics of infection in the changing world. Clin Microbiol Infect. 2011; 17: 494-501. 
13. Bajani MD, Ashford DA, Bragg SL, Woods CW, Aye T, Spiegel RA, et al. Evaluation of four commercially available rapid serologic tests for diagnosis of leptospirosis. J Clin Microbiol. 2003; 41: 803-809.

14. Courdurie C, Le Govic Y, Bourhy P, Alexer D, Pailla K, Theodose R, et al. Evaluation of different serological assays for early diagnosis of leptospirosis in Martinique (French West Indies). PLoS Negl Trop Dis. 2017; 11: e0005678.

15. Sakka V, Tsiodras S, Giamarellos Bourboulis EJ, Giamarellou H. An update on the etiology and diagnostic evaluation of a leukemoid reaction. Eur $\mathbf{J}$ Intern Med. 2006; 17; 394-8.

16. De Silva NL, Niloofa M, Fernando N, Karunanayake L, Rodrigo C, De Silva HJ, et al. Changes in full blood count parameters in leptospirosis: a prospective study. Int Arch Med. 2014; 7 : 1-4.
17. N Kharidehal, R Prasad. Peripheral Blood Leukemoid Reaction: An Unusual Presentation of Leptospirosis. The Internet Journal of Infectious Disease. 2005. Available at: https://ispub.com/IJID/5/2/3596 Fecha de acceso: $20 \mathrm{de}$ febrero de 2017.

18. Monterrubio Villar J, González Velasco C, Cidoncha Calderon B, Cidoncha Gallego M. Colecistitis alitiasica como forma de presentación de una leptospirosis grave. Cir Esp. 2013; 94: 264-265.

19. Pajarola M, Hartmann G. A case of Weil's disease with leukemoid reaction and fatal course. Schweiz Med Wochenschr. 1977; 107: 1855- 1856.

20. Hill JM, Duncan CN. Leukemoid reactions. Am J Med Sci. 1941; 201: 847.

21. Velasco Castrejón O, Rivas Sánchez B, Gutiérrez E, Chávez L, Duarte P, Chavarria S, et al. Leptospira ¿simulador o causante de leucemia? Rev Cubana Med Trop 2005; 57: 17-24. 